\title{
Warum die Bundesregierung erneut verfassungsriskante Regelbedarfe vorlegt
}

Die Höhe der Grundsicherung für erwerbsfähige Hilfebedürftige war seit Einführung von Hartz IV umstritten. Spätestens mit der Forderung des Bundesverfassungsgerichts, die Regelsätze transparenter zu berechnen, verbanden sich vielfach Hoffnungen auf substanzielle Verbesserungen. Sie wurden in weiten Teilen enttäuscht. Hat der Gesetzgeber seinen Gestaltungsspielraum überschritten, indem er sich von dem vorrangigen Ziel leiten ließ, die Höhe der Regelbedarfe nicht wesentlich zu erhöhen? Und welche Gründe gab es dafür?

\section{Vorgeschichte}

Mit Urteil vom 9. Februar 2010 hat das Bundesverfassungsgericht (BVerfG) aus dem Menschenwürdepostulat des Artikel 1 Grundgesetz (Art. 1 GG) und dem Sozialstaatsprinzip des Art. 20 GG ein Grundrecht auf Gewährleistung eines menschenwürdigen Existenzminimums abgeleitet. In den Leitsätzen der Entscheidung heißt es weiter, dass dieses Grundrecht der Konkretisierung und stetigen Aktualisierung durch den Gesetzgeber bedürfe, der die zu erbringenden Leistungen an dem jeweiligen Entwicklungsstand des Gemeinwesens und den bestehenden Lebensbedingungen auszurichten habe. Dabei stehe ihm ein Gestaltungsspielraum zu. Das BVerfG stellte jedoch insbesondere an die Ermittlung des Existenzminimums dezidierte Anforderungen: Dieses sei in einem transparenten und sachgerechten Verfahren realitätsgerecht sowie nachvollziehbar auf der Grundlage verlässlicher Zahlen und schlüssiger Berechnungsverfahren zu bemessen. Ein im Oktober 2010 vorgelegter Gesetzentwurf der Bundesregierung, mit dem die Regelbedarfe der erwachsenen Leistungsempfänger zum 1.1.2011 um $5 €$ erhöht werden sollten, scheiterte am 17.12.2010 am Veto des Bundesrates. Nach schwierigen, acht Wochen dauernden Verhandlungen hat am 25.2.2011 das Gesetz zur Ermittlung von Regelbedarfen und zur Änderung des Sozialgesetzbuches Zweites Buch (SGB II) und des Sozialgesetzbuches Zwölftes Buch (SGB XII) sowohl Bundesrat als auch Bundestag passiert. Der erzielte Kompromiss enthält zwar einige veritable Verbesserungen, eine Erhöhung der Regelbedarfe konnte jedoch nicht erreicht werden. Die nach wie vor bestehenden verfassungsrechtlichen Bedenken der rot-grünen Opposition im Vermittlungsausschuss fanden schließlich Eingang in einen neuen $\$ 10$ des Regelbedarfs-Ermittlungsgesetzes (RBEG), in dem vorsorglich die zentralen Probleme aufgelistet werden und der Auftrag erteilt wird, diese bis zur Durchführung der nächsten Einkommens- und Verbrauchsstichprobe im Jahr 2013 und bis zum Inkrafttreten der neuen Regelbedarfe im Jahr 2015 einer Lösung zuzuführen. Das BVerfG hat in seinem Urteil vom 9.2.2011 aber nicht den geringsten Zweifel daran gelassen, dass der Gesetzgeber zum 1.1.2011 verfassungskonforme Regelsätze vorlegen muss und nicht erst vier Jahre später. Im Folgenden werden zunächst die gravierendsten verfassungsrechtlichen Probleme des im Februar dieses Jahres verabschiedeten Gesetzes kurz dargestellt (Abschnitt 2). Diese Übersicht ersetzt nicht eine vertiefende verfassungsrechtliche Auseinandersetzung, wie sie an anderer Stelle bereits geleistet wurde und worauf verwiesen werden kann (Münder 2011; Lenze 2011, S. 1104ff.). Sie dient vielmehr als Grundlage für das Hauptanliegen dieses Beitrages: die mutmaßlichen Motive der Bundesregierung wie auch die gesellschaftspolitische Tragweite des gefundenen Hartz-IV-Kompromisses auszuleuchten (Abschnitt 3).

\section{Die verfassungsrechtlichen Risiken}

Der Gesetzgeber hat an verschiedenen Stellschrauben gedreht, um sein wichtigstes Ziel zu erreichen, nämlich auf keinen Fall die Regelsätze erhöhen zu müssen:
(1) So berechnet er den Regelbedarf der erwachsenen Leistungsberechtigten nicht mehr anhand der Verbrauchsausgaben der untersten $20 \%$, sondern an den Ausgaben der untersten $15 \%$ der nach ihren Nettoeinkommen geschichteten Einpersonenhaushalte (Abschnitt 2.1).

(2) Außerdem hat er - entgegen der ausdrücklichen Vorgabe des BVerfG - die Gruppe der verdeckt Armen nicht aus der Referenzgruppe herausgerechnet (Abschnitt 2.2).

(3) Schließlich behandelt er Eltern wie Alleinstehende, weil dies ebenfalls Kosten spart (Abschnitt 2.3)

(4) Ferner hat er eine Vielzahl von Ausgaben als nichtregelsatzrelevant eingestuft (Abschnitt 2.4).

(5) Und nicht zuletzt erweckt der für Kinder ermittelte Regelbedarf schwerwiegende Bedenken, da die der Berechnung zugrunde liegenden Daten zum großen Teil auf nicht aussagekräftigen Stichproben basieren (Abschnitt 2.5).

Es spricht einiges dafür, dass in der Gesamtschau mit den neuen Regelbedarfen ein menschenwürdiges Existenzminimum nicht gewährleistet werden kann. 


\subsection{STATISTIK-METHODE}

Das BVerfG hatte festgestellt, dass die auf der Einkommens- und Verbrauchsstichprobe (EVS) basierende Statistik-Methode, die das sozialrechtliche Existenzminimum an den Verbrauchsausgaben der untersten $20 \%$ der nach ihrem Nettoeinkommen geschichteten Haushalte orientiert, für die Berechnung des Regelbedarfs grundsätzlich geeignet sei (BVerfG, 9.2.2010, $1 \mathrm{BvL}$ 1/09 u.a., Rn. 168 = NJW 2010, S. 505). In dem RBEG hat der Gesetzgeber jedoch bei der Ermittlung des Regelbedarfs der Erwachsenen nicht - wie bisher - auf die nach ihrem Einkommen geschichteten untersten $20 \%$, sondern nur noch auf die untersten $15 \%$ der Einpersonenhaushalte abgestellt. Damit ist der obere Grenzwert der betrachteten Referenzeinkommen von $990 €$ auf $901 €$ gesunken. Es ist durchaus möglich, dass das BVerfG diese Vorgehensweise im Fall einer erneuten Befassung mit der Frage akzeptieren würde, weil auch die $15 \%$-Basis noch repräsentative und statistisch valide Daten zur Verfügung stellt. Sollte das BVerfG dies absegnen, dann ist jedoch im Gegenzug davon auszugehen, dass die übrigen im Urteil vom 9.2.2010 entwickelten verfassungsrechtlichen Anforderungen umso schärfer geprüft werden, wenn schon am Anfang des Berechnungsverfahrens eine Stellschraube zulasten der Leistungsberechtigten angezogen wurde. Summieren sich restriktive Vorgehensweisen, so besteht letztendlich die Gefahr, dass das menschenwürdige Existenzminimum nicht mehr garantiert werden kann. Dass dieses nicht nur in Relation zu den Verbrauchsausgaben der einkommensschwächsten Haushalte zu bestimmen ist, sondern dass im Hintergrund auch ein objektiver Maßstab bereitstehen muss, deutet sich in der Formulierung des BVerfG an, wenn es einschränkend darauf hinweist, dass der Einsatz der Statistik-Methode nur vertretbar sei unter der „Prämisse, dass auch das Ausgabeverhalten unterer Einkommensgruppen der Bevölkerung zu erkennen gibt, welche Aufwendungen für das menschenwürdige Existenzminimum erforderlich sind“ (BVerfG, 9.2.2010, 1 BvL $1 / 09$ u.a., Rn. 166). Es wäre deshalb rechtspolitisch sinnvoll, im Rahmen einer Ergebniskontrolle anhand eines Warenkorbes zu überprüfen, ob denn die Referenzgruppe ihrerseits ein als objektiv gesetztes Existenzminimum decken kann. Nicht von ungefähr hatten die Wohlfahrtsverbände dem Übergang von der Warenkorb- hin zur Statistikmethode Anfang der 1990er Jahre nur unter der Voraussetzung einer regelmäßigen Gegenkontrolle zugestimmt (vgl. Sartorius 2000, S. 90f.).

\subsection{VERDECKTE ARMUT}

Wenn der Gesetzgeber die Referenzgruppe auf die untersten $15 \%$ der nach ihrem Einkommen geschichteten Einpersonenhaushalte reduziert, dann stellt sich das Problem der verdeckten Armut in ungleich schärferer Weise als in dem Sachverhalt, der dem Urteil des BVerfG vom 9.2.2010 zugrunde lag. Das BVerfG hatte den Gesetzgeber darauf verpflichtet, entsprechend seiner Pflicht zur Fortentwicklung seines Bedarfsermittlungssystems, „bei der Auswertung künftiger Einkommens- und Verbrauchsstichproben darauf zu achten, dass Haushalte, deren Nettoeinkommen unter dem Niveau der Leistungen nach dem Sozialgesetzbuch Zweites Buch und dem Sozialgesetzbuch Zwölftes Buch inklusive der Leistungen für Unterkunft und Heizung liegt, aus der Referenzgruppe ausgeschieden werden." Dem ist der Gesetzgeber nicht nachgekommen und er kann sich auch nicht darauf zurückziehen, dass dies nicht möglich gewesen wäre. In der öffentlichen Anhörung von Sachverständigen vor dem Ausschuss für Arbeit und Soziales am 22.11.2010 haben sowohl der Vertreter des Institutes für Arbeitsmarktund Berufsforschung (IAB) als auch die Vertreterin des Statistischen Bundesamtes auf Nachfrage erklärt, dass die verdeckte Armut aus den Daten der EVS 2008 durchaus hätte herausgenommen werden können, wenn ein entsprechender Auftrag der Bundesregierung vorgelegen hätte. Bereits am 1.12.2010 lag eine Ausarbeitung des Statistischen Bundesamtes im Auftrag der Fraktion DIE LINKE vor, in dem alle Einpersonenhaushalte aus der EVS herausgerechnet worden waren, die weniger als den Betrag von $639 €$ zur Verfügung hatten - dies entsprach dem durchschnittlichen Regelsatz und den durchschnittlichen Kosten der Unterkunft des Jahres 2008. Auf der Grundlage der Verbräuche der untersten $20 \%$ der Haushalte ergab sich ein fortgeschriebener Regelbedarf für das Jahr 2011 in Höhe von $392 €$ (Ausschussdrucksache 17(11)361, 1). Es ist zu konzedieren, dass der Gesetzgeber im Rahmen seiner Gestaltungsfreiheit verschiedene Wege gehen kann, die Gruppe der verdeckt Armen abzugrenzen. Dass er dies auch tun muss, ist nach der Entscheidung des BVerfG vom 9.2.2010 klar. Zumindest die rotgrüne Opposition sieht in diesem Punkt ein verfassungsrechtliches Risiko, denn im Vermittlungsausschuss wurde zuletzt ein zusätzlicher $\$ 10$ RBEG aufgenommen, der die Bundesregierung verpflichtet, bis zum 1.7.2013 einen unter Mitwirkung des Statistischen Bundesamtes und von Sachverständigen zu erstellenden Bericht vorzulegen, der u.a. die Frage der verdeckten Armut aufklären soll. Es ist jedoch kaum vorstellbar, dass das BVerfG es hinnimmt, dass die Leistungsberechtigten damit noch einmal fünf Jahre warten müssen, ehe ein verfassungsgemäßer Regelbedarf in Kraft tritt.

\subsection{BEHANDLUNG VON ELTERN WIE ALLEINSTEHENDE}

Verfassungsrechtliche Irritationen ruft auch die Entscheidung des Gesetzgebers hervor, bei der Ermittlung des Regelbedarfs Eltern wie Alleinstehende zu behandeln. Zwar werden in $\$ 4$ des RBEG die Einpersonenhaushalte, bei denen die untersten $15 \%$ der in der EVS 2008 ermittelten Haushalte zugrunde gelegt werden, den Familienhaushalten gegenübergestellt, für die auf die Gruppe der untersten $20 \%$ der Haushalte abgestellt wird. Im Weiteren ergibt sich dann aber, dass auch die Regelbedarfe der Erwachsenen in Familienhaushalten auf der Grundlage der Verbrauchsausgaben der untersten $15 \%$ der Einpersonenhaushalte berechnet werden. Das BVerfG ist auf diesen Punkt nicht eingegangen, weil bis Ende 2010 die Regelleistung aller Leistungsberechtigten nach den Verbrauchsausgaben der $20 \%$-Referenzgruppe ermittelt wurde. Es hat aber festgestellt, dass bei der Ermittlung der für einen Alleinstehenden notwendigen Leistungen die „Beschränkung auf Einpersonenhaushalte sachgerecht" sei (BVerfG, 9.2.2010, 1 BvL 1/09 u.a., bverfg. de, Rn. 168). Offengelassen wurde die Frage, ob dies auch für Mehrpersonenhaushalte und Familien zu gelten hat. Die Behandlung von Eltern als Alleinstehende ist jedoch willkürlich und kann nicht begründet werden. Alleinstehende in dieser niedrigen Einkommensschicht gehören zu einer besonders deklassierten Teilgruppe der Gesellschaft, deren Einkommen und Ausgaben keine Rückschlüsse auf die Bedarfe von Erwachsenen in Mehrpersonen- 
Haushalten zulassen. Dies war von den Sachverständigen Irene Becker und Rudolf Martens im Verfahren vor dem hessischen Landessozialgericht (LSG), das zur Vorlage vor das BVerfG geführt hatte, dezidiert dargelegt worden (LSG HE, 29.10.2008, L 6 AS 336/07, juris, Rn. 108 ff.). Die Berechnung der Leistungen für Kinder auf der Grundlage einer $20 \%$-Referenzgruppe und die für Eltern auf Grundlage einer $15 \%$-Referenzgruppe ist zudem methodisch inkonsequent: Der Bemessung des kindlichen Existenzminimums liegt eine Aufteilung von Haushaltsausgaben auf Kind und Eltern unter Berücksichtigung von Haushaltsgrößenersparnissen zugrunde. Letztere sind nur gerechtfertigt, wenn die den Eltern zugerechneten Fixkosten tatsächlich gedeckt sind. Dies ist infolge der Bezugnahme auf den anderen Referenzhaushaltstyp der Alleinstehenden nicht gewährleistet (Ausschussdrucksache 17(11)309, 113 (Becker)). Da die EVS alle Daten über die Verbrauchsausgaben der nach ihrem Nettoeinkommen geschichteten untersten $20 \%$ der Familienhaushalte bereitstellt, ist nicht zu erklären, dass die Bedarfe der Erwachsenen in Familienhaushalten nicht ebenfalls daran zu bemessen sind. Kinder leben mit ihren Eltern in einem Haushalt - die Familienmitglieder teilen denselben Lebensstandard. Stattdessen wird eine ökonomische Einheit (die Familie) aufgesplittet und der Bedarf der einen auf der Grundlage von $20 \%$ der Familienhaushalte, der Bedarf der anderen auf der Grundlage von 15 \% der Einpersonenhaushalte berechnet, ohne dies auch nur ansatzweise zu begründen. Die Ableitung des Bedarfes von Eltern anhand der Verbrauchsausgaben von Alleinstehenden verstößt gegen Art. 6 Abs. 1 GG, weil die Pflicht zur Förderung der Familie besonders den wirtschaftlichen Zusammenhalt der Familie umfasst (BVerfGE 61, S. 18ff.; 62, S. 323ff.; 75, S. 382ff.). Außerdem ist ein Verstoß gegen Art. 3 Abs. 1 GG zu konstatieren, weil er Ungleiches gleichbehandelt und nicht seiner Eigenart nach verschieden (BVerfGE 108, S. 52ff.; 103, S. 242ff.). Auch in Bezug auf diese Problematik findet sich in $\$ 10$ Abs. 2 Nr. 3 RBEG die Absichtserklärung, für künftige Datenerhebungen der EVS „Vorschläge zur Weiterentwicklung“ der Ermittlung der Verbrauchsausgaben von Erwachsenen in Mehrpersonenhaushalten vorzulegen. Auch hier stellt sich die Frage, warum erst weitere fünf Jahre ins Land gehen müssen, bevor ein verfas- sungsgemäßer Zustand erreicht ist - zumal die wesentlichen Daten bereits jetzt alle vorliegen.

\subsection{NICHT REGELSATZRELEVANTE BEDARFE}

Das BVerfG hat ausgeführt, dass es verfassungsrechtlich nicht $\mathrm{zu}$ beanstanden sei, dass die in den einzelnen Abteilungen der Einkommens- und Verbrauchsstichprobe erfassten Ausgaben „des untersten Quintils nicht vollständig, sondern als regelleistungsrelevanter Verbrauch nur zu einem bestimmten Prozentsatz in die Bemessung der Regelleistungen einfließen. Allerdings muss der jeweilige Abschlag sachlich gerechtfertigt sein" (BVerfG, 9.2.2010, 1 BvL 1/09 u.a., Rn. 170). Der Gesetzgeber hat zwar auf prozentuale Abschläge verzichtet, er hat stattdessen aber wiederholt von der Möglichkeit Gebrauch gemacht, durchschnittliche Ausgaben der Referenzgruppe für nicht regelsatzrelevant zu erklären. Es kann selbstverständlich darüber gestritten werden, ob Ausgaben für Schnittblumen, Haustiere, Gartengeräte, Küchenuhren oder die Kosten der chemischen Reinigung zum menschenwürdigen Existenzminimum gehören. Dass die Leistungsberechtigten hier unterschiedliche Entscheidungen treffen, ist jedoch gerade ein Charakteristikum der Statistik-Methode. Der Gesetzgeber hat mit seiner Vorgehensweise Statistik- und Warenkorbmethode willkürlich vermischt (Becker 2010, S. 10ff.). Aus den statistisch ermittelten Verbrauchsausgaben der untersten $15 \%$ bzw. $20 \%$ der Haushalte nimmt er - in umgekehrter Anwendung des Warenkorbmodells - wieder eine Vielzahl von Waren aus dem Korb heraus, die als nicht relevant für den Regelbedarf eingestuft werden. Obwohl das BVerfG dies ausdrücklich gebilligt hat, hat es doch an anderer Stelle ausgeführt, dass dem Statistikmodell die Überlegung zugrunde liege, dass der individuelle Bedarf eines Hilfebedürftigen in einzelnen Ausgabepositionen vom durchschnittlichen Verbrauch abweichen kann, der Gesamtbetrag der Regelleistung es aber ermöglichen solle, „einen überdurchschnittlichen Bedarf in einer Position durch einen unterdurchschnittlichen Bedarf in einer anderen auszugleichen. Der Gesetzgeber muss deshalb die regelleistungsrelevanten Ausgabepositionen und Beträge so bestimmen, dass ein interner Ausgleich möglich ist" (BVerfG,
9.2.2010, 1 BvL 1/09 u.a., Rn. 172). Diese Voraussetzung könnte aber dann nicht mehr erfüllt sein, wenn an einer Vielzahl von Ausgabepositionen gekürzt wird und damit der Regelbedarf insgesamt zu tief sinkt (Münder 2011, S. 28). Öffentlich debattiert wurden die Abschläge in Höhe von $19 €$ durch die Herausnahme der Ausgaben für Tabak und Alkohol, wobei Letztere durch preiswertes Mineralwasser aus dem Diskounter in Höhe von monatlich $2,99 €$ ersetzt wurden. Was bei den Stammtischen (sic!) vielleicht gut ankommt, bedeutet aber, dass die Ermittlung der Regelbedarfe davon ausgeht, dass ein Grundsicherungsempfänger nicht einmal an einem Abend im Monat zuhause mit Freunden ein Bier trinkt. Ein Mindestmaß an sozialer Teilhabe ist damit nicht möglich. Da im unteren Einkommensbereich kaum Sparvermögen gebildet wird, verteilt sich bei „enthaltsamen“ Haushalten der nicht ausgegebene Posten für alkoholische Getränke und Tabak auf andere Ausgabenpositionen. Wenn der Gesetzgeber den Grundsicherungsempfängern schon eine gesunde Lebensführung vorschreiben will, dann hätte er im Sinne der Substitutionstheorie auch nur das Ausgabeverhalten der Haushalte betrachten dürfen, die abstinent leben. Wer sein Geld nicht für Alkohol und Tabak ausgibt, hat vielleicht höhere Kosten für Sportgeräte, Bücher oder Ernährung (Martens 2010a, S. 12). Dem Gesetzgeber wurde zwar ein größerer Gestaltungsspielraum für den Bereich der Teilhabe am sozialen Leben zugestanden, wenn dieser aber soweit ausgedünnt ist, dass selbst verbreitete Formen der Geselligkeit gar nicht mehr enthalten sind, könnte hier eine Grenze überschritten sein. Für die Gruppe der untersten $15 \%$ der Alleinstehenden summieren sich die Kürzungen aufca. $135 €$, dies entspricht $27 \%$ der Ausgaben der Referenzgruppe (Frankfurter Arbeitskreis Armutsforschung 2010, S. 4). Der Herausnahme nicht regelsatzrelevanter Positionen kommt somit die Funktion zu, die früher die Abschläge hatten: Sie bieten die Möglichkeit, genau den Regelbedarf zu „berechnen“, der arbeitsmarktpolitisch und fiskalisch als tragbar erscheint. So ist es wohl kein statistischer Zufall, dass monatelanges Rechnen genau den Betrag von $364 €$ ergab, den schon im Jahr 2008 der Siebente Existenzsicherungsbericht als Existenzminimum für das Jahr 2010 in Aussicht gestellt hatte (BT-Drs. 16/11065, S. 7). 


\subsection{REGELBEDARF FÜR KINDER}

Verfassungsrechtliche Bedenken sind auch gegenüber dem neuen Regelbedarf für Kinder angebracht. Während für die Erwachsenen nur auf die Ausgaben der untersten $15 \%$ der nach ihrem Einkommen geschichteten Haushalte abgestellt wurde, wurden für den Regelbedarf von Kindern die untersten $20 \%$ der Haushalte zugrunde gelegt, was darin begründet liegt, dass ansonsten keine statistisch ausreichend große Gruppe zustande gekommen wäre. Das Ergebnis der konkreten Ermittlung des Kinderbedarfs ist einigermaßen überraschend: Danach sollen die alten - ins Blaue hinein geschätzten Kinderregelsätze - zu hoch gewesen sein, und zwar zunehmend mit steigendem Alter: Bei den null bis sechs Jahre alten Kindern um $2 €$, bei den sieben bis 14 Jahre alten Kindern um $9 €$ und bei den 14 bis 18 Jahre alten Kindern um $12 €$ (\$ 8 RBEG). Zwar werden die Sätze nicht gekürzt, allerdings werden die Leistungen für Kinder solange nicht angepasst, bis die rechnerische Lücke durch Inflation und Preisentwicklung geschlossen ist (\$\$77 IV, 134 SGB II). Dieses Ergebnis widerspricht den in den letzten Jahren von Wohlfahrtsverbänden vorgelegten Berechnungen, wonach die Kinderregelsätze zu niedrig seien (Martens et al. 2009; Neue Caritas spezial 10/2008). Auch hatten Untersuchungen ergeben, dass der im Sozialgeld für Lebensmittel vorgesehene Betrag für Kinder $a b$ sieben Jahren keine ausgewogene Ernährung ermöglicht (Kersting/Claußen 2010; Heinz 2010, S. 73). Um zu verifizieren, ob die Familien der Referenzgruppe für ihre Kinder überhaupt selber ein menschenwürdiges Existenzminimum sicherstellen können, würde es sich anbieten, anhand der Warenkorbmethode zu überprüfen, ob es der Regelbedarf erlaubt, Kinder und Jugendliche angemessen zu ernähren und zu kleiden. Eine Erklärung dafür, dass die Diskrepanz zum früheren Regelsatz insbesondere bei den Jugendlichen so hoch sei, lässt sich jedoch unschwer darin finden, dass der Gesetzgeber neuerdings für die Jugendlichen ab dem 15. Lebensjahr unterstellt, dass sie im selben Maß Alkohol und Tabak konsumieren wie Erwachsene und ihnen hierfür ebenfalls ca. $16 €$ vom Regelbedarf abzieht. Diese Vorgehensweise entspricht wieder einer vom BVerfG ausdrücklich verbotenen „Schätzung ins Blaue hinein“, denn solange diese Annahme nicht empirisch belegt ist, hat sie nach den Vorgaben des BVerfG für ein transparentes, realitätsgerechtes und auf belastbaren Daten beruhendem Verfahren zu unterbleiben. Jüngste Veröffentlichungen der Bundeszentrale für gesundheitliche Aufklärung belegen außerdem den gegenteiligen Trend: Der Anteil der 12- bis 17-Jährigen, die wenigstens ein Mal in der Woche Alkohol trinken, ist danach auf $13 \%$ (FAZ, 5.2.2011, S. 9), der Anteil der rauchenden Jugendlichen auf 12,5\% gesunken (FAZ, 25.2.11, S. 9).

Gravierend ist außerdem, dass bei der Neuberechnung der Kinderregelsätze viele Verbrauchspositionen der EVS nicht mit Zahlen unterlegt sind, sondern Leerstellen aufweisen, was daran liegt, dass zu einzelnen Ausgabeposten Fallzahlen von weniger als 25 Haushalten vorhanden waren. Es sind aber mindestens 100 Haushalte erforderlich, um einen Fehlerquotienten von unter $10 \%$ zu erreichen (Ausschuss für Arbeit und Soziales, Protokoll 17/41, S. 658 (Statistisches Bundesamt)). Damit wird das Verfahren zur Ermittlung der Regelsätze für Kinder der verfassungsgerichtlich geforderten Obliegenheit zur Transparenz nicht gerecht. Die neu ermittelten Regelsätze der Kinder sind auch deshalb niedriger als die alten ,gegriffenen“ Beträge, weil der Regelbedarf von Kindern um diejenigen Aufwendungen gekürzt wurde, die ab dem 1.1.2011 über den Bildungs- und Teilhabebedarf des $\$ 28$ SGB II gedeckt werden sollen. Dem persönlichen Schulbedarf von $100 €$ im Jahr gem. $\$ 28$ Abs. 3 SGB II stehen Kürzungen von monatlich $8,33 €$ gegenüber (Ausschussdrucksache 17(11)309, S. 115 (Becker)). Das Schulbedarfspaket, das bislang zusätzlich zur Regelleistung gewährt wurde, ist also nicht so großzügig wie es erscheint, es wird langfristig nahezu vollständig mit stagnierenden Regelbedarfen der Kinder gegenfinanziert, die auf dem jetzigen Niveau verharren werden, selbst wenn die regelbedarfsrelevanten Preise steigen werden. Auch dem monatlichen Betrag in Höhe von $10 €$ zur Finanzierung der Teilhabe am sozialen und kulturellen Leben in der Gemeinschaft stehen Kürzungen des Regelbedarfs von Kindern in Höhe von monatlich $1,08 €$ bei den 6- bis 14-Jährigen und monatlich $3,58 €$ bei den 14- bis 18-Jährigen gegenüber (Becker ebd.). Während die Teilhabeleistungen nur auf Antrag und für bestimmte Aktivitäten gewährt werden, betrifft die damit begründete Regelbedarfskürzung alle Kinder. Sollten die $10 €$ pro Kind flä- chendeckend abgerufen werden, so wäre dies wohl hinnehmbar, obwohl darauf hinzuweisen ist, dass durch die Gegenfinanzierung der finanzielle Aktionsradius für diejenigen Kinder schrumpft, die keinen Sport treiben und kein Musikinstrument erlernen möchten. Erste Ergebnisse belegen aber, dass die als Sachleistungen ausgestalteten Angebote des Bildungsund Teilhabepakets des $\$ 28$ SGB II von der Mehrheit der Kinder und Jugendlichen nicht nachgefragt wird. In den Städten haben ca. $27 \%$, in den Landkreisen $30 \%$ der 2,5 Mio. Berechtigten die erforderlichen Anträge gestellt (Deutsche Presse Agentur vom 28.6.2011). Die zögerliche Nachfrage lässt sich zum einen darauf zurückführen, dass Gutscheine erfahrungsgemäß vor allem von denjenigen Leistungsberechtigten genutzt werden, die ihr Leben ohnehin gut organisieren können (Sell 2011, S. 32), während Menschen mit verschiedenen Integrationshindernissen weniger von ihnen profitieren. Zum anderen war aber absehbar, dass die Beschränkung der Förderung auf institutionell organisierte Angebote vor allem an den Bedürfnissen älterer Kinder und Jugendlicher nach Individualität und Selbstbestimmung vorbeigeht, da diese ihre Identität oft in Peer Groups entwickeln und ihren Interessen allein oder mit Freunden nachgehen. Wenn aber die Mehrheit der Minderjährigen die Angebote des Bildungs- und Teilhabepaketes nicht in Anspruch nimmt, ist von einer verfassungsrechtlich relevanten Unterdeckung ihres Bedarfes auszugehen, da die Regelbedarfe um die idealtypisch von $\$ 28$ SGB II abgedeckten Positionen gekürzt wurden. Der Gesetzgeber hätte die Probleme, die mit dem Antragserfordernis verbunden sind, antizipieren müssen. Eine Herausnahme von Positionen aus dem Regelbedarf von Kindern und Jugendlichen hätte erst erfolgen dürfen, nachdem sich die Deckung der Teilhabe-Bedarfe durch Sachleistungen als erfolgreich herausgestellt hätte.

In der Gesamtschau ist der Gesetzgeber ein verfassungsrechtliches Risiko eingegangen, indem er die Regelbedarfe für Erwachsene und Kinder derartig „auf Kante genäht" hat. Im Kontext des steuerrechtlichen Existenzminimums hatte das BVerfG genau dies kritisiert und ein - vorsorgliches oder kompensierendes - Überschreiten der Mindestwerte für geboten gehalten (BVerfG 10.11.1998-2 BvL 42/93 - BVerfGE 99, S. 246-263). Dies muss erst 
recht im Grundsicherungsrecht gelten, denn die Leistungsbezieher leben allein auf der Grundlage der Mindestbeträge, während im Steuerrecht den Steuerpflichtigen regelmäßig ein höherer Betrag zum Leben verbleibt.

\section{Warum die Bundesregie- rung bewusst verfassungs- rechtliche Risiken eingeht}

Ehernes Ziel der Reform war, dass es keine signifikante Erhöhung der Regelsätze geben durfte. Es können dabei fiskalische, politische, ideologische und strukturelle Motive unterschieden werden.

\subsection{FISKALISCHE GRÜNDE}

Die fiskalischen Gründe sind offensichtlich: Die Erhöhung der Regelsätze hätte sowohl Mehrausgaben im Etat des Arbeits- und Sozialministeriums als auch Mindereinnahmen im Etat des Finanzministeriums bedeutet, weil im Steuerrecht das freizustellende Existenzminimum der Erwachsenen ebenfalls hätte erhöht werden müssen. Die Bundesregierung spielt mit den verfassungsriskanten Regelbedarfen auf Zeit: Wenn sich kein erstinstanzliches Sozialgericht findet, das die Frage dem BVerfG direkt vorlegt, kann es bis zu fünf Jahre und länger dauern, bis sich Leistungsbezieher durch den gesamten Instanzenweg geklagt haben und das BVerfG erneut entscheidet. Vielleicht spekuliert die Regierung darauf, dass sie höhere Leistungen dann wieder nicht rückwirkend zahlen muss und sogar eine weitere Umsetzungsfrist erhält. In diesen Jahren, die unterdessen ins Land gehen, lassen sich immerhin Milliardenbeträge sparen. Die Missachtung der Vorgaben des BVerfG kann sich also „lohnen“. Bei den fiskalischen Erwägungen gibt es auch eine Interessenübereinstimmung von Bundesregierung und Bundesländern, weil Letztere höhere Regelbedarfe für die in ihrer Zuständigkeit liegenden erwerbsunfähigen Bezieherinnen und Bezieher von SGB-XIILeistungen zahlen müssten.

\subsection{POLITISCHES KALKÜL}

Neben den rein fiskalischen Gründen lässt sich auch ein politisches Eigeninteresse der Regierung für die vehemente Weigerung ausmachen, die Regelsätze zu erhöhen. Eine signifikante Erhöhung hätte nämlich die angeblichen Erfolge der Bundesregierung im Kampf gegen Armut und Arbeitslosigkeit zunichte gemacht, weil mit einem Schlag das Ausmaß des Niedriglohnsektors sichtbar geworden wäre: Berechnungen des IAB aus dem Jahr 2008 hatten ergeben, dass bei einer angenommenen Erhöhung des Regelbedarfes auf $420 €$ bis zu 2 Mio. zusätzliche Hartz-IV-Empfänger zu verzeichnen seien und Mehrkosten von $10 \mathrm{Mrd}$. $€$ anfallen würden (Feil/Wiemers 2008). Dies sollte aber auf gar keinen Fall geschehen.

\subsection{IDEOLOGISCHE GRÜNDE}

Es kamen aber noch gewichtige ideologische Gründe hinzu. Denn auch eine materielle Verbesserung der Lage der Leistungsbezieher, die ohne eine explizite Erhöhung der Regelsätze ausgekommen wäre, konnte nicht durchgesetzt werden. So hatte die rot-grüne Opposition im Bundesrat vorgeschlagen, die Aufwendungen für selten anfallende Verbrauchspositionen, wie z.B. Kühlschränke, Waschmaschinen und Fahrräder, aus dem Regelbedarf herauszunehmen und wieder als einmalige Leistungen zu gewähren; ähnliches sollte für Familien mit hohen Mobilitätskosten gelten. Dies hätte nicht zu einer Ausweitung des Kreises der Leistungsberechtigten geführt und hätte sich auch deshalb angeboten, weil sich diese Verbrauchsausgaben in der EVS ohnehin nicht realitätsgerecht und transparent erfassen lassen: Die entsprechenden Positionen in der Statistik enthalten Leerstellen, da zu wenige Haushalte in dem dreimonatigen Befragungszeitraum diese Anschaffungen getätigt hatten und deshalb hierzu kein valides Datenmaterial vorliegt.

Wichtige ideologische Motive müssen auch deshalb vorhanden sein, weil sogar dort, wo es effektiv $z u$ Verbesserungen des Leistungsniveaus kam, diese von keiner der Parteien des Vermittlungsausschusses nach außen kommuniziert wurden. Die neuen Begründungs- und Transparenzanforderungen des BVerfG hatten nämlich zur Folge gehabt, dass das Licht auf Stellen fiel, die bislang kaschiert worden sind. So hatte der Gesetzgeber einfach unterstellt, dass in den Regelsätzen die Kosten für die Warmwassererzeugung enthalten seien und den Leistungsberechtigten deshalb einen bestimmten Betrag von den Energiekosten im Rahmen der Kosten der Unterkunft wieder abgezogen (BT-Drs. 16/1410, 23). Grundlegende Kritik an dieser Vorgehensweise hatte das Sächsische LSG in seiner Entscheidung vom 29.3.2007 vorgetragen, indem es mit allen zur Verfügung stehenden Ermittlungsmethoden akribisch nachgewiesen hat, dass in der Regelleistung des SGB II Haushaltsenergie zur Warmwasserbereitung nicht enthalten sein konnte (L 3 AS 101/06). Das Bundessozialgericht (BSG) hatte diese Einwände in seiner Entscheidung vom 27.2.2008 mit den Sätzen abgetan, die Festlegung der Regelleistung sei letztendlich ein „normativ/wertender Prozess, der in seinen einzelnen Schritten keinen naturwissenschaftlich-mathematisch ableitbaren Richtigkeitsansprüchen unterliegt“" (B 14/11b AS 15/07 R, juris, Rn. 22). Diese Auffassung war jedoch nach der Entscheidung des BVerfG vom 9.2.2010 nicht mehr haltbar, denn seitdem muss der Gesetzgeber zur Ermittlung des Regelbedarfs alle existenznotwendigen Aufwendungen in einem transparenten und sachgerechten Verfahren realitätsgerecht sowie nachvollziehbar auf der Grundlage verlässlicher Zahlen und schlüssiger Berechnungsverfahren" bemessen (BVerfG, 3. Leitsatz). Schließlich schaltete sich im Rahmen der Verhandlungen des Vermittlungsausschusses zur Neuregelung der Regelbedarfe im Januar 2011 der Landkreistag ein mit dem Hinweis, dass die Kosten der Warmwasserzubereitung nicht im Regelbedarf enthalten seien (FAZ 6.1.2011, 9). Im Verlaufe der Verhandlungen des Vermittlungsausschusses ist daher ein Kompromiss dahingehend gefunden worden, dass die Kosten der Warmwasserzubereitung nunmehr zusätzlich zum Regelbedarf erbracht werden. Diese Neuregelung führt faktisch zu einer Erhöhung des Regelbedarfs für einen alleinstehenden Leistungsberechtigten in Höhe von $6,88 €$, einem Paar in Höhe von 15,08 € und weiteren Erhöhungen für jedes zusätzliche Familienmitglied.

Wenn diese indirekte Erhöhung der Leistungen öffentlich nicht kommuniziert wurde, dann liegt der Schluss nahe, dass gegenwärtig verfassungskonforme Regelsätze nicht mehr vermittelbar erscheinen. Der höchst emotional geladene öffentliche Diskurs über den deutschen Sozialstaat nach der Verkündung des Urteils vom 9.2.2010 hat die Stimmung im Land erheblich verändert. Die Medien spielten 
hierbei eine nicht unerhebliche Rolle. Waren vor Urteilsverkündung $48 \%$ der Bevölkerung der Meinung, die Grundsicherungsleistungen seien zu niedrig, und nur $7 \%$, sie seien zu hoch (Stern-Umfrage, 28.10.2009), so sprechen sich ein Jahr später $56 \%$ gegen eine Erhöhung aus, $14 \%$ sind gar für eine Kürzung und nur $36 \%$ halten eine Anhebung der Sätze für erforderlich (Emnid, 26.9. 2010). Neben den Äußerungen des deutschen Außenministers („spätrömische Dekadenz“, „leistungsloser Wohlstand“) war es vor allem der Werbefeldzug der Arbeits- und Sozialministerin von der Leyen, mit dem zwei Sätze unaufhörlich in die Hirne der Bevölkerung gefräst wurden: So hat die Ministerin zum einen mit der Diskussion um die Bildungschipcard strategisch die öffentliche Aufmerksamkeit von den wesentlichen Vorentscheidungen in Bezug auf die Regelsätze abgelenkt und mit ihrem Mantra, dass die „Leistung bei den Kindern ankommen müsse“ den Subtext verbreitet, dass Eltern im SGB-II- und SGB-XII-Bezug nicht vertrauenswürdig seien und das ihren Kinder zugedachte Geld für sich selber verwenden würden, ein Vorurteil, das bar jedweder empirischer Evidenz ist. Das zweite Leitmotiv lautete, „dass sich Leistung lohnen müsse" und die Friseuse und die Verkäuferin am Ende mehr Geld in der Tasche haben müssten als die Leistungsbezieher. Das Lohnabstandsgebot, das hier angesprochen wird, ist aber seit der Entscheidung des BVerfG zumindest verfassungsrechtlich obsolet, weil in jedem Fall stets das menschenwürdige Existenzminimum gedeckt sein muss. Folgerichtig wurde es stillschweigend aus $\$ 28$ SGB XII entfernt. Es ist aber politisch nach wie vor hoch virulent. Niedrige Regelleistungen sind ein Kernstück der Hartz-Gesetzgebung, die im Übrigen so erfolgreich war, dass selbst die Bertelsmann-Stiftung, die federführend bei der Entwicklung der Agenda 2010 mitgewirkt hat (Spindler 2007), nun ihrerseits warnend darauf hinweist, dass der deutsche Arbeitsmarkt die stärkste Deregulierung in Europa und einen im internationalen Vergleich besonders hohen Anteil an atypischer Beschäftigung aufweist (Eichhorst et al. 2010, S. 9). Arbeitnehmerinnen und Arbeitnehmer wissen heute, dass der Verlust des Arbeitsplatzes in einem mittleren Lebensalter den endgültigen Absturz bedeuten und nach einem Jahr des Bezuges von Arbeitslosen- geld I sich die in Jahrzehnten aufgebaute materielle und berufliche Lebensstellung in kürzester Zeit verflüchtigen kann (Lenze 2010, S. 142). Die Angst vor dem HartzIV-Absturz hat sowohl Beschäftigte wie Arbeitsuchende „konzessionsbereiter", sprich billiger, werden lassen. In den zwei Jahren nach Einführung der HartzGesetzgebung brachen die Lohnkosten je produzierter Einheit in Deutschland um „unheimliche fast vier Prozent ein, nachdem sie bis 2005 gestiegen waren " (Financial Times Deutschland, 12.03.2010, S. 27). Dass die Bundesregierung im Vermittlungsausschuss zu vielen Kompromissen bereit war, sich jedoch erfolgreich gegen eine Erhöhung der Regelbedarfe zur Wehr gesetzt hat, zeigt, dass ein niedriges Niveau an Grundsicherungsleistungen in Deutschland weiterhin als das notwendige Gegenstück zum politisch gewollten Niedriglohnsektor fungiert.

\subsection{UMVERTEILUNG}

Schließlich führt die Nichterhöhung der Regelbedarfe strukturell die Strategie der Bundesregierung einer Umverteilung von unten nach oben fort. Hier ist der Zeitpunkt der Verkündung des Urteils und seiner Umsetzung von Bedeutung. Die Entscheidung des BVerfG vom 9.2.2010 erfolgte zwar nach der Banken- und Wirtschaftskrise 2008, jedoch zeichnete sich die wirtschaftliche Erholung bereits deutlich ab. Die Umsetzung des Urteils erfolgte nach der sogenannten Euro-Rettung, mit der sich die Bundesrepublik weiter erheblich verschuldet hat. Es besteht offensichtlich Einigkeit dahingehend, die Verursacher der Krise - allen voran Banken und Versicherer - nicht wesentlich an den Kosten der Rettungsaktionen zu beteiligen. Das Haushaltsbegleitgesetz 2011 war deshalb angetreten, den Bundeshaushalt bis 2014 um knapp 20 Mrd. $€$ zu entlasten. Das Gros der Einsparungen wird bei den einkommensschwächsten Mitgliedern der Gesellschaft realisiert, bei denen am wenigsten Widerstand erwartet wird. Abgesehen von der kaum nennenswerten Erhöhung der Regelbedarfe der Erwachsenen werden bei den Leistungsbeziehern im Wege des Haushaltsbegleitgesetzes 2011 insgesamt jährlich 4,9 Mrd. $€$ eingespart (BGBl. 2010, Teil I Nr. 63, 1885 ff.): So entfällt u.a. der befristete Zuschlag nach Bezug von Arbeitslosengeld (Alg) I nach $\$ 24$ SGB II; außerdem entrichtet der Bund nicht länger die Beiträge für die versicherungspflichtigen Bezieher von Alg II nach $\$ 170$ I Nr. 1 SGB VI; nicht zuletzt entfällt durch die Änderung der Einkommensanrechnung des $\$ 10$ Abs. 5 S. 1 und 2 BEEG (Gesetz zum Elterngeld und zur Elternzeit) das Elterngeld für Leistungsbezieherinnen, die bis zur Geburt und darüber hinaus vollständig auf Grundsicherung angewiesen sind - eine Regelung, die im Übrigen verfassungsrechtlich nicht haltbar sein dürfte (Lenze 2011, S. 3ff.). Außerdem sollen pro Jahr weitere 2 Mrd. $€$ durch den „flexiblen Einsatz" der aktiven Arbeitsförderung sprich durch Kürzungen - eingespart werden. An weiteren Einsparungen zulasten der Ärmsten ist durch den Kompromiss des Vermittlungsausschusses nun noch hinzugekommen, dass die Entlastung der Kommunen durch weitere Einsparungen bei der Bundesagentur für Arbeit gegenfinanziert werden muss, was dort eine Lücke von fast $10 \mathrm{Mrd}$. $€$ bis 2015 reißen wird und durch Kürzungen bei den Arbeitslosen kompensiert werden muss. Und dies in einer Situation, in der die ungeheure Verwendung von Steuermitteln zur Stützung von Teilen der Wirtschaft zeigt, dass der Staat noch nicht in der Situation ist, „sich wegen der leeren Haushaltskassen notgedrungen gegen eine wirksame Armutsbekämpfung entscheiden zu müssen“ (Rothkegel 2010, S. 144).

Hinzu kommt, dass die Spargesetze auch die vom Grundgesetz intendierte Gleichwertigkeit der Lebensverhältnisse gem. Art. 72 II GG beeinträchtigen werden, da zwar auch einige Ballungsgebiete im Westen betroffen sind, vor allem aber der Osten Deutschlands sehr einseitig belastet wird (Martens 2010b). Auch wenn es sich um eine vergleichsweise weich formulierte politische Zielsetzung mit sehr begrenztem Regelungsgehalt handelt (Osterloh 2002, S. 312), die zudem keine subjektiv-rechtlichen Ansprüche Einzelner enthält, so ist es doch erklärtes Ziel von Art. 3 I i.V.m. Art. 20 I GG, gleichwertige Lebensverhältnisse i.S. des Art. 72 II GG für alle Bürger zu eröffnen (BVerfG, 22.11.2000, 1 BvR 239/94 u.a. = BVerfGE 102, 254, 312). Das Haushaltsbegleitgesetz 2011 bewirkt jedoch in Verbindung mit dem prioritären Ziel der Bundesregierung, den Regelbedarf von Erwachsenen und Kindern auf keinen Fall nennenswert zu erhöhen, eine Vertiefung der Kluft zwischen Ost und West. 
Becker, I. (2010): Bedarfsbemessung bei Hartz IV. Zur Ableitung von Regelleistungen auf der Basis des "Hartz-IV-Urteils" des Bundesverfassungsgerichts, Wiso Diskurs, Oktober

Eichhorst, W./Marx, P./Thode, E. (2010): Atypische Beschäftigung und Niedriglohnarbeit - Benchmarking Deutschland: Befristete und geringfügige Tätigkeiten, Zeitarbeit und Niedriglohnbeschäftigung, Gütersloh Feil, M./Wiemers, J. (2008): Höheres ALG II und Kindergrundsicherung: Teure Vorschläge mit erheblichen Nebenwirkungen, IAB-Kurzbericht (11)

Frankfurter Arbeitskreis Armutsforschung (2010): Menschenwürde, Teilhabe und die scheinbare Objektivität von Zahlen, Fachliche Stellungnahme des Frankfurter Arbeitskreises Armutsforschung zum Entwurf für ein Gesetz zur Ermittlung von Regelbedarfen und zur Änderung des Zweiten und Zwölften Buches Sozialgesetzbuch (Regelbedarfs-Ermittlungsgesetz - RBEG)

Heinz, D. (2010): Einige Überlegungen zu den die Existenz sichernden Leistungen für die Kosten der Ernährung bei Kindern, zu möglicher Fehlernährung infolge zu geringer Leistungen und zu möglichen rechtlichen Konsequenzen, in: Zeitschrift für das Fürsorgewesen 62 (4), S. 73-78 Kersting, M./Clausen, K. (2007): Wie teuer ist eine gesunde Ernährung für Kinder und Jugendliche?, in: ErnährungsUmschau 54 (9), S. 508-513

Lenze, A. (2010): Abschied von der Solidargemeinschaft, in: Kritische Justiz 43 (2), S. 132-144

Lenze, A. (2011): Die Streichung des Elterngeldes für Grundsicherungsempfängerlnnen - ein gleichheitsrechtliches Desaster! , in: info also 29 (1), S. 3-9

Lenze, A. (2011): Sind die neuen Hartz-IV-Sätze verfassungskonform?, in: Neue Zeitschrift für Verwaltungsrecht (NVwZ) 30 (18), S. $1104 f f$. Martens, R. (2010a): Die Regelsatzberechnungen der Bundesregierung sowie der Vorschlag des Paritätischen Gesamtverbandes für bedarfsdeckende Regelsätze, Expertise vom 22.10.
Martens, R. (2010b): Unter unseren Verhältnissen II. Atlas der Sozialkürzungen, hrsg. vom Paritätischen Gesamtverband, Berlin Martens, R./zur Gathen, M./Schneider, U./Rock, J./ Stilling, G./ Struck, N. (2009): Zur Bestimmung eines bedarfsgerechten Existenzminimums für Kinder nach dem Statistikmodell gemäß § 28 SGB XII (Sozialhilfe), in: Der Paritätische Gesamtverband (Hrsg.): Was Kinder brauchen ...Für eine offene Diskussion über das Existenzminimum für Kinder nach dem Statistikmodell gemäß § 28 SGB XII (Sozialhilfe), Berlin, S. XI-41

Münder, J. (2011): Entspricht der Regierungsentwurf eines Gesetzes zur Ermittlung von Regelbedarfen und zur Änderung des Zweiten und Zwölften Buches Sozialgesetzbuch vom 20.10.2010 den verfassungsrechtlichen Anforderungen der Entscheidung des Bundesverfassungsgerichts 1 BvL 1/09 vom 9.2.2010 - Eine rechtsgutachterliche Stellungnahme, in: Spellbrink, W. (Hrsg.): Verfassungsrechtliche Probleme des SGB II, München, S. 15-50

Osterloh, L. (2002), Der verfassungsrechtliche Gleichheitssatz - Entwicklungslinien der Rechtsprechung des Bundesverfassungsgerichts, in: Europäische Grundrechte Zeitschrift (EuGRZ) 29 (13-14), S. 309-313 Rothkegel, R. (2010): Ein Danaergeschenk für den Gesetzgeber. Zum Urteil des Bundesverfassungsgerichts vom 9. Februar 2010-1 BvL 1, 3, 4/09, in: Zeitschrift für Sozialhilfe und SGB (ZFSH/SGB) 49 (3), S. $135-146$

Sell, S. (2011): Teilhabe und Bildung als Sachleistungen: bisherige Erfahrungen mit Gutschein- und Chipkartensystemen, in: Archiv für Wissenschaft und Praxis der sozialen Arbeit 42 (1), S. 24-37

Spindler, H. (2007): War auch die Hartz-Reform ein Bertelsmann Projekt?, in: Wenicke, J./Bultmann, T. (Hrsg.): Netzwerk der Macht Bertelsmann, Marburg, S. 243-276

Sartorius, U. (2000): Das Existenzminimum im Recht, Baden-Baden 